\title{
Síntese e caracterização de peneiras moleculares mesoporosas tipo MCM-41 a partir de diferentes direcionadores estruturais
}

\author{
Manoel Marcelino da Silva; \\ Alanna Oliveira Cortez; \\ J oana Maria de Farias Barros ${ }^{3}$.
}

\begin{abstract}
${ }^{1}$ Universidade Federal Rural do Semi-Árido / Pós-Graduando (Mestrado) em Ambiente, Tecnologia e Sociedade - e-mail: manoelmarcelinodasilva@gmail.com

2 Universidade Federal Rural do Semi-Árido / Pós-Graduando (Mestrado) em Ambiente, Tecnologia e Sociedade - e-mail: alanna_cortez@hotmail.com

3 Universidade Federal de Campina Grande / Centro de educação e Saúde - Professora adjunta email: joanamfb@ufcg.edu.br
\end{abstract}

Resumo: Neste trabalho a peneira molecular mesoporosa tipo MCM-41 foi sintetizada a partir de diferentes direcionadores estruturais visando a obtenção de materiais nanoestruturados com propriedades catalíticas e estabilidades hidrotérmicas para aplicações específicas. Os materiais foram sintetizados pelo método hidrotérmico a $100^{\circ} \mathrm{C}$ por 120 horas, apresentando, todas as amostras, no gel de síntese a seguinte composição molar: 1,00 Surfactante: 4,00 $\mathrm{SiO}_{2} ; 1,00 \mathrm{Na}_{2} \mathrm{O}: 200,00 \mathrm{H}_{2} \mathrm{O}$. Os materiais resultantes, após calcinação a $500^{\circ} \mathrm{C}$ por 2 horas, foram caracterizados por: difração de raiosX (DRX), análise termogravimétrica (TG/DTG), espectroscopia na região do infravermelho (FT-IR) e área superficial pelo método de BET. As análises de DRX dos catalisadores indicaram que os materiais apresentaram estrutura hexagonal característica do material mesoporoso tipo MCM-41. As curvas TG mostraram que a decomposição do direcionador estrutural ocorre nos materiais em temperaturas inferiores a $550^{\circ} \mathrm{C}$. As amostras apresentaram variações quanto à área superficial específica, diâmetro médio de poros e espessura da parede de sílica, em função do direcionador estrutural.

Palavras-chave: Peneiras moleculares; MCM-41; Síntese hidrotérmica; Direcionador estrutural.

\section{I ntrodução}

As peneiras moleculares foram definidas em 1932 (FLANIGEN, 1991), e por definição tem-se que são sólidos capazes de adsorver moléculas de determinados tamanhos de acordo com o diâmetro de seus poros. Assim, a International Union of Pure and Applied Chemistry (IUPAC) classifica os materiais porosos de acordo com o tamanho de seus poros em microporosos (menor que $2 \mathrm{~nm}$ ), mesoporosos (de 2 a $50 \mathrm{~nm}$ ) e macroporosos (maior que $50 \mathrm{~nm}$ ). A presença desses poros exerce influência nas propriedades físicas dos materiais, como por exemplo, em sua densidade, estabilidade térmica e resistência mecânica. Sendo assim o controle desses poros é de extrema importância comercial e industrial. 
Para síntese dos materiais mesoporosos tipo MCM-41, se faz necessário o uso de um surfactante (direcionador estrutural) para que o sistema de poros seja formado de maneira a replicar forma e a organização que este surfactante se encontra. A síntese original do MCM-41 foi realizada em água e meio alcalino, com utilização de um surfactante iônico como direcionador estrutural, formando um material orgânico-inorgânico ordenado (KRESG et. al.,1992). O MCM-41 possui poros em formato cilíndrico e uma estrutura hexagonal. As paredes desses poros são formadas por sílica amorfa, são relativamente finas, com espessuras entre 0,6 a 1,2 $\mathrm{nm}$. As paredes entre os poros destes materiais têm uma espessura de aproximadamente $1 \mathrm{~nm}$, como ilustra a figura 1.

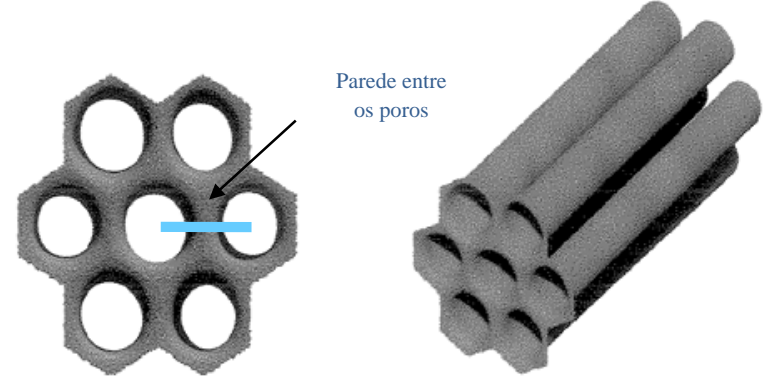

Figura 1: Esquema estrutural dos poros do MCM-41 (SCHUTH, 1995).

Para que a caracterização do MCM-41 seja realmente confiável, faz-se necessário o uso de três técnicas, difração raio X microscopia eletrônica de transmissão e análise de adsorção. Este trabalho tem como objetivo principal a síntese hidrotérmica da sílica mesoporosa denominada MCM-41 a partir de diferentes direcionadores estruturais.

\section{Materiais e Métodos}

\subsection{Síntese da peneira molecular MCM-41}

O material MCM-41 foi sintetizado através do método hidrotérmico usando sílica gel, silicato de sódio, direcionador estrutural e água. Estes reagentes foram adicionados em proporções estequiométricas de modo a se obter um gel com composição molar: 1,00 CTMABr: 4,00 $\mathrm{SiO}_{2}$ : 1,00 $\mathrm{Na}_{2} \mathrm{O}: 200,00 \mathrm{H}_{2} \mathrm{O}$. Para a obtenção do gel, adicionou-se sílica (Merck) ao silicato de sódio (Vetec) e à metade da água requerida para a síntese. $\mathrm{O}$ sistema foi submetido à agitação por 2 horas a $60^{\circ} \mathrm{C}$, em seguida uma solução aquosa do direcionador (CTMABr) (Vetec) mais a outra metade da água foi adicionada à mistura que permaneceu sob agitação por mais 1 hora em temperatura ambiente. Após o preparo do gel, este foi transferido para um vaso de teflon, posto em uma autoclave de aço inoxidável e aquecido em estufa a $100^{\circ} \mathrm{C}$ por 120 horas (5 dias). A cada 24 horas foi feita a correção do pH do gel para a faixa entre 9,5 a 10,0 com uma solução 30\% de ácido acético. Após 96 horas com o pH já estável foi adicionado acetato de sódio numa proporção molar direcionador/sal igual a 3 para a completa estabilização da sílica. Em seguida o sistema permaneceu por mais 24 horas na estufa a $100^{\circ} \mathrm{C}$. Após a cristalização a autoclave foi retirada da estufa e resfriada até a temperatura ambiente. O seu conteúdo foi lavado com água destilada e em seguida com uma solução $2 \%$ de ácido clorídrico em etanol para a remoção de parte do surfactante. Finalmente, o sólido resultante do processo de cristalização foi submetido à estufa a $100^{\circ} \mathrm{C}$ por 12 horas. O mesmo procedimento foi seguido para a peneira molecular sintetizada com o tetradeciltrimetilamônio (TDTMABr) (Vetec). Este procedimento experimental está representado esquematicamente na figura 2 . 


| Fonte de sílica

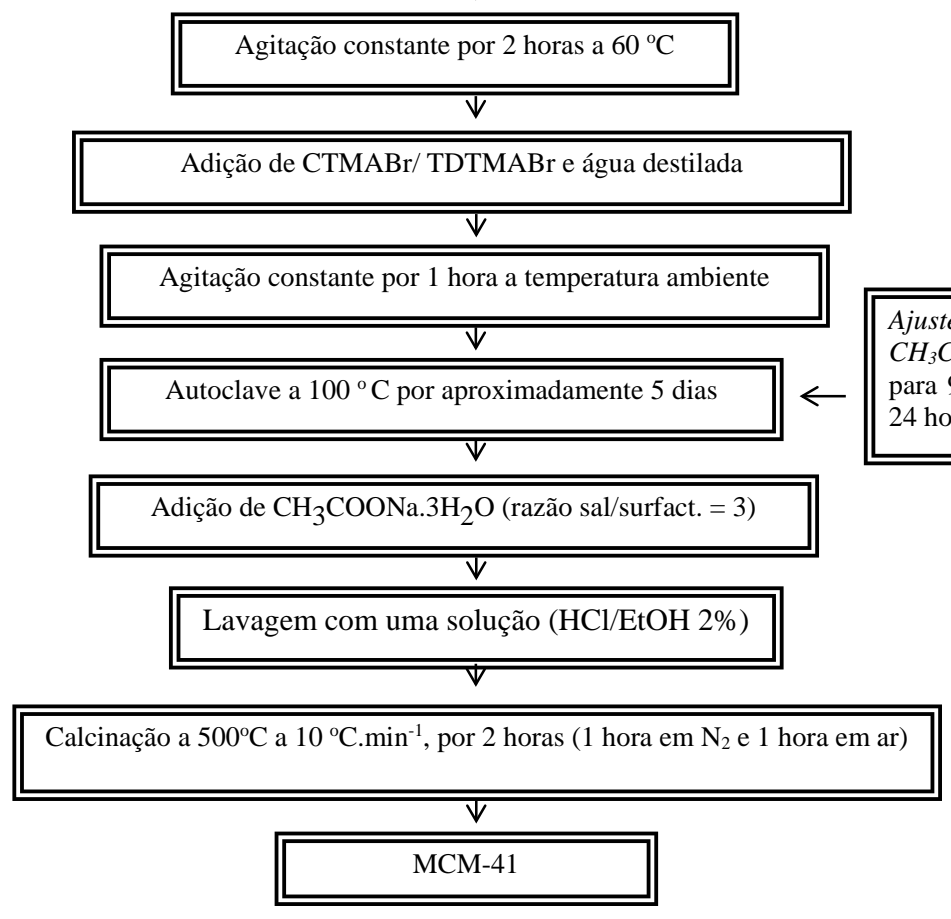

Figura 2. Fluxograma das etapas de síntese dos materiais MCM-41.

As amostras sintetizadas receberam a seguinte nomenclatura: MCM-41 sintetizada com brometo de cetiltrimetilamônio - MCM-41/CTMABr e MCM-41 sintetizada com brometo de tetradeciltrimetilamônio MCM-41/ TDTMABr.

\section{2 - Calcinação do Material}

O sistema utilizado para este processo está representado na figura 3. A etapa de calcinação desta série de catalisadores ocorreu em duas etapas onde inicialmente a amostra foi submetida a uma rampa de aquecimento de $10^{\circ} \mathrm{C} \mathrm{min}{ }^{-1}$ da temperatura ambiente até $500^{\circ} \mathrm{C}$ em atmosfera inerte de nitrogênio a uma vazão de $100 \mathrm{~mL} \mathrm{~min}^{-1}$. Após ter atingido a temperatura de $500^{\circ} \mathrm{C}$, o sistema permaneceu nesta condição por 1 hora. Em seguida, o fluxo de nitrogênio foi trocado por ar sintético na vazão de $100 \mathrm{~mL} \mathrm{~min}^{-1}$ por tempo adicional de 1 hora. Este processo de calcinação visa a remoção do direcionador estrutural dos poros dos catalisadores. 


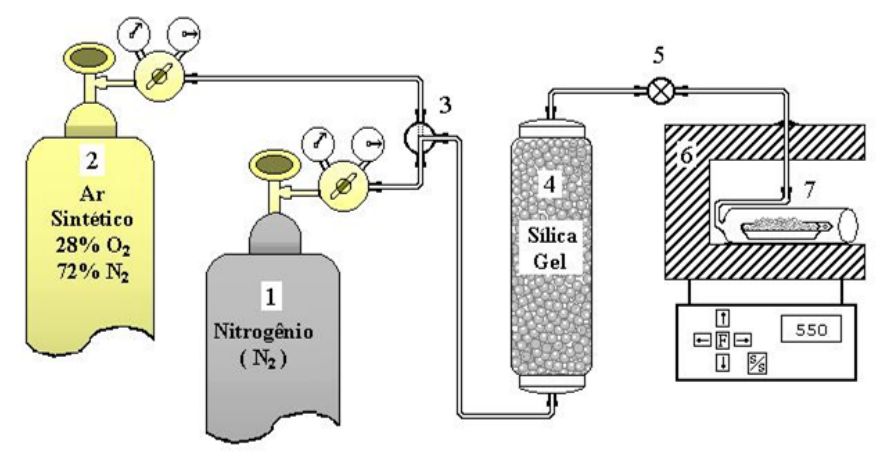

Figura 3. Diagrama esquemático do sistema utilizado para a calcinação das amostras. Onde: 1 e 2 - cilindros de $\mathrm{N}_{2}$ e ar sintético respectivamente, 3 - válvula de seleção de gases, 4 - adsorvente para retenção de umidade, 5 válvula de ajuste de vazão, 6 - forno e 7 - amostra.

\section{3 - Caracterização das peneiras mesoporosas}

\subsection{1 - Difração de raios - $X$}

As amostras foram caracterizadas por difração de raios X (DRX) em um equipamento da Shimadzu modelo XRD-6000 utilizando-se uma fonte de radiação de CuKa com voltagem de $30 \mathrm{kV}$, corrente de $30 \mathrm{~mA}$ e filtro de $\mathrm{Ni}$. Os dados foram coletados na faixa de $2 \theta$ de 1 a 10 graus com velocidade de goniômetro de $2^{\circ} \mathrm{min}^{-1}$ com um passo de 0,02 graus.

Pelo difratograma de raios-X do material mesoporoso MCM-41 observa-se a presença de três reflexões que são representados em sua forma simplificada pelas reflexões dos planos (100), (110) e (200).

Os difratogramas de raios $\mathrm{X}$ foram utilizados para a identificação da estrutura hexagonal característica dos materiais mesoporosos tipo MCM-41. O parâmetro do arranjo hexagonal mesoporoso $\mathrm{a}_{0}$ (parâmetro de rede) da estrutura MCM-41 é obtido através do pico de reflexão para o plano (100), o qual é o mais característico no difratograma de raios-X.

\subsection{2 - Espectroscopia na região do infravermelho}

Os espectros de absorção na região do infravermelho foram obtidos em um espectrofotômetro de infravermelho por transformada de Fourier da Bomem modelo MB 102, usando KBr como agente dispersante. As pastilhas foram preparadas pela mistura de aproximadamente $0,7 \mathrm{mg}$ de amostra com uma quantidade suficiente de $\mathrm{KBr}$ para se atingir a concentração de $1 \%$ em massa. Em seguida, a mistura foi homogeneizada em um almofariz, transferida para o empastilhador e submetida a uma pressão de 8 ton $\mathrm{cm}^{-2}$, formando uma pastilha fina e translúcida. Os espectros foram obtidos na região de 4000 a 400 $\mathrm{cm}^{-1}$.

\subsection{3 - Análise termogravimétrica}


As análises termogravimétricas (TG/DTG) dos materiais em estudo foram realizadas em uma termobalança da Mettler Toledo TGA/SDTA 851, a uma taxa de aquecimento de $10{ }^{\circ} \mathrm{C} \mathrm{min}{ }^{-1}$, na faixa de temperatura ambiente até $900^{\circ} \mathrm{C}$, utilizando-se atmosfera dinâmica de nitrogênio na vazão de $25 \mathrm{~mL}$ $\min ^{-1}$. Em todas as análises foram utilizados cadinhos de alumina de $70 \mu \mathrm{L}$ e uma massa de amostra de aproximadamente $15 \mathrm{mg}$.

A partir das curvas termogravimétricas das amostras antes da etapa de calcinação, foram determinadas as quantidades de água e de direcionador orgânico presentes (CTMABr/TDTMABr), bem como, as faixas de temperatura onde estas moléculas foram removidas. Estes dados foram fundamentais para a determinação da menor temperatura de calcinação necessária para a remoção do direcionador orgânico dos poros dos materiais.

\section{3 - Resultados e Discussões}

\section{1 - Difração de raios - X}

As propriedades estruturais das amostras MCM-41/CTMABr e MCM-41/TDTMABr pura foram caracterizadas por medidas de DRX. O resultado desta análise está apresentado na figura 4 . O padrão de DRX da amostra apresenta três picos típicos, um com uma elevada intensidade, atribuída a linha de reflexão do plano (100) e dois outros com menor intensidade atribuídos às reflexões dos planos (110) e (200) característicos da estrutura hexagonal mesoporosa como descrito por Beck et al. (1992)

a)

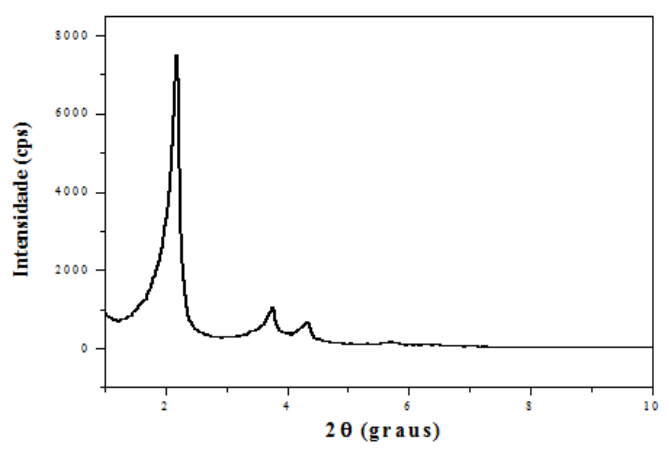

b)

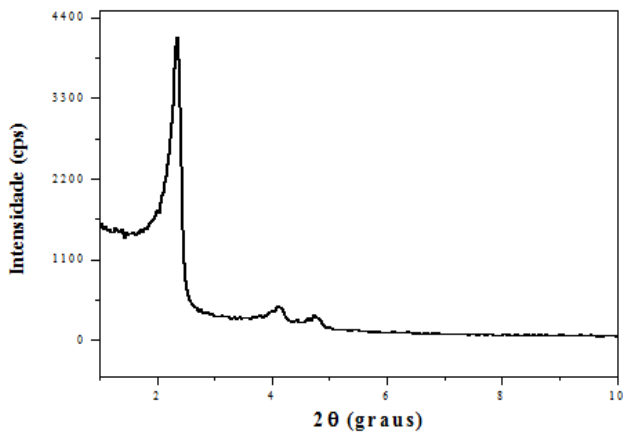

Figura 4 - a) Difratograma de raio-X da amostra MCM-41/CTMABr calcinada a $500^{\circ} \mathrm{C}$. b) Difratograma de raio$\mathrm{X}$ da amostra MCM-41/TDTMABr calcinada a $500^{\circ} \mathrm{C}$.

Um pico em $2 \theta=2,19 \mathrm{~nm}$, correspondente à reflexão (100) na amostra MCM-41/CTMABr, nos fornece, de acordo com a regra de Bragg, a dimensão do parâmetro de rede hexagonal $\left(\mathrm{a}_{0}\right)$ para o MCM41 de 4,65nm. Na amostra MCM-41/TDTMABr houve uma contração da rede, indicando uma interação diferente entre a fonte de sílica e o direcionador estrutural. A tabela 1 apresenta os resultados dos parâmetros relativos à difração de raios-X. 
Tabela 1 - Parâmetros relativos à difração de raios - X das amostras

\begin{tabular}{lcccc}
\hline Amostra & $\mathbf{2 \theta}$ & $\mathbf{k h l}$ & $\mathbf{d ~ ( n m )}$ & $\mathbf{a}_{\mathbf{0}}(\mathbf{n m})$ \\
\hline MCM-41/CTMABr & 2,19 & $(100)$ & 4,03 & 4,65 \\
MCM-41/ TDTMABr & 2,37 & $(100)$ & 3,73 & 4,31 \\
\hline
\end{tabular}

\section{2 - Espectroscopia na região do infravermelho}

O espectro de absorção na região do infravermelho do material mesoporoso MCM-41/CTMABr está apresentado na figura 5, correspondendo à região espectral entre 4000 e $400 \mathrm{~cm}^{-1}$.

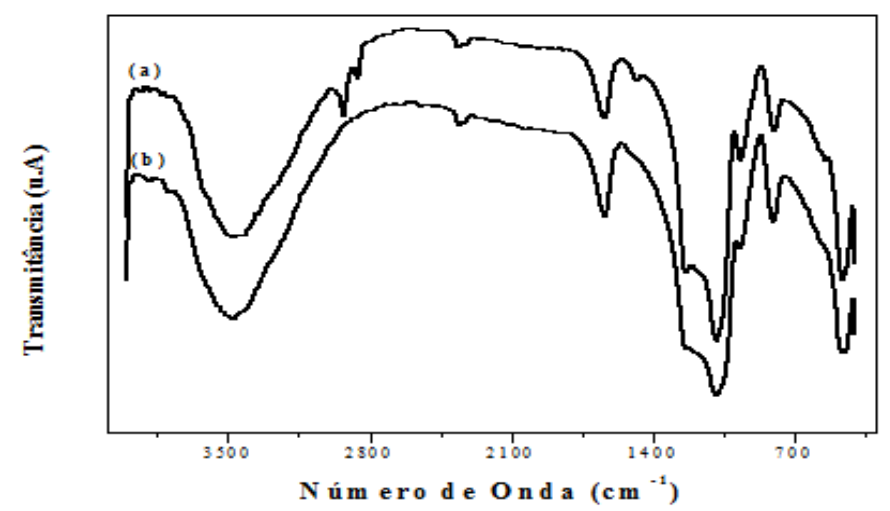

Figura 5: Espectros de infravermelho da amostra MCM-41: (a) amostra não calcinada e (b) amostra calcinada

Os espectros da amostra MCM-41/CTMABr antes da etapa de calcinação, apresentam uma banda de absorção em 2930 e $2849 \mathrm{~cm}^{-1}$ correspondente a moléculas o surfactante. A ausência desta banda nos espectros do material calcinado evidencia a completa remoção do direcionador orgânico das estruturas originais.

A figura 6 mostra as isotermas de adsorção/ dessorção de $\mathrm{N}_{2}$ a $77 \mathrm{~K}$. todas as amostras apresentam isotermas do tipo IV de acordo com a classificação da IUPAC, com baixa adsorção a pressões relativas $<0,1$. A partir dos resultados desta análise foi possível determinar a área superficial específica pelo método de BET, o diâmetro médio dos poros através do método BJH e a espessura da parede de sílica de acordo com a Equação 1. Estes dados estão apresentados na tabela 2.

$$
\text { wt }=\mathrm{a}_{\mathrm{o}}-\mathrm{d}_{\mathrm{p}} . \quad \text { (Equação 1) }
$$

Onde: $w t=$ Espessura da parede de sílica; $\mathrm{a}_{\mathrm{o}}=$ Parâmetro de rede e $\mathrm{d}_{\mathrm{p}}=$ Diâmetro de poro. 
a)

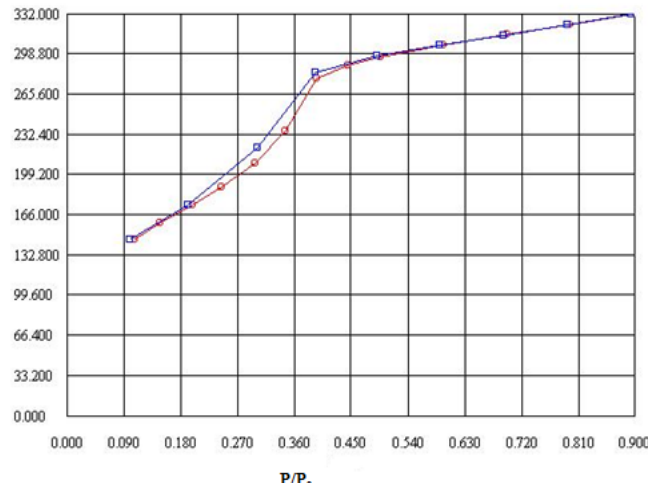

$\mathrm{P} / \mathrm{P}$ 。 b)

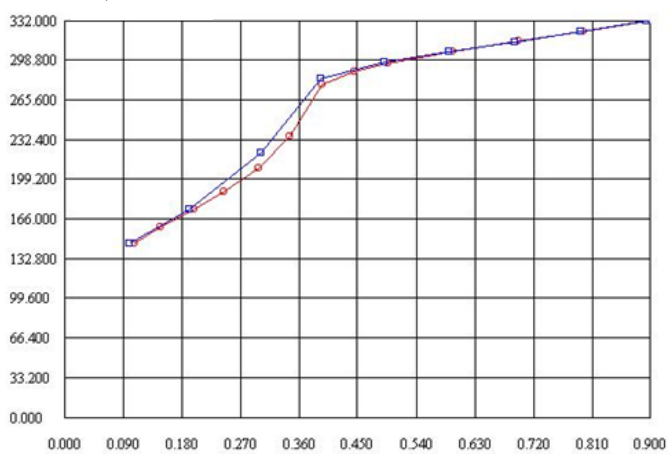

P/P.

Figura 6 - a) Isotermas de adsorção/dessorção de $\mathrm{N}_{2}$ a 77K da amostra MCM-41/CTMABr calcinada; b) Isotermas de adsorção/dessorção de $\mathrm{N}_{2}$ a 77K da amostra MCM-41/TDTMABr calcinada.

Tabela 2 - Propriedades estruturais das amostras

\begin{tabular}{cccc}
\hline Amostra & $\begin{array}{c}\text { Área superficial } \\
\text { específica BET }\left(\mathbf{m}^{\mathbf{2}} \mathbf{~ g}^{-\mathbf{1}}\right)\end{array}$ & $\begin{array}{c}\text { Diâmetro médio dos } \\
\text { poros (nm) }\end{array}$ & $\begin{array}{c}\text { Espessura da parede de } \\
\text { sílica (nm) }\end{array}$ \\
\hline $\mathrm{MCM}-41 / \mathrm{CTMABr}$ & 668 & 3,07 & 1,58 \\
$\mathrm{MCM}-41 / \mathrm{TDTMABr}$ & 550 & 3,13 & 1,18 \\
\hline
\end{tabular}

\section{3 - Análise termogravimétrica}

As análises termogravimétricas das amostras sintetizadas na forma não calcinada, obtidas em atmosfera de nitrogênio, estão apresentadas na figura 7.

De acordo com dados da literatura (KRUK, 2000) a análise termogravimétrica do material mesoporoso do tipo MCM-41 apresenta três perdas de massa principais, são feitas as seguintes atribuições para estes eventos: Dessorção de água fisicamente adsorvida; Decomposição do surfactante e Condensação de grupos silanóis. 
a)

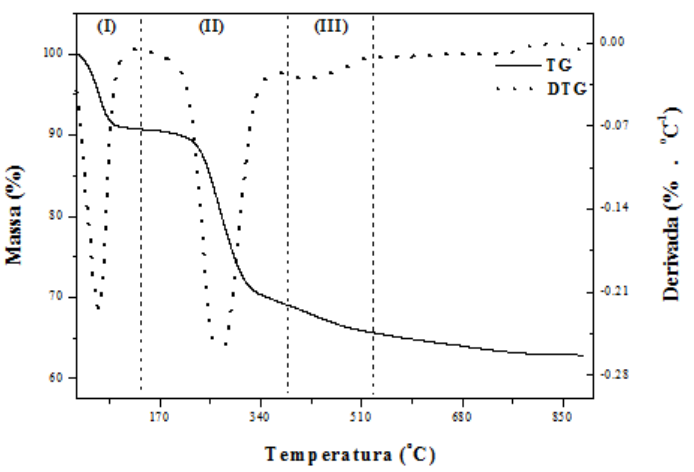

b)

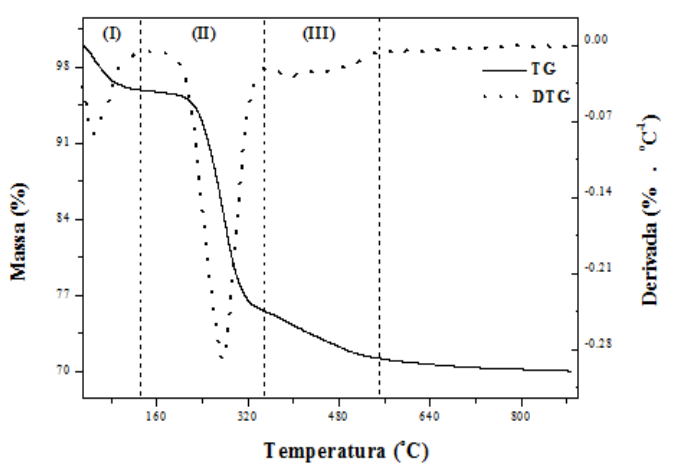

Figura 7 - a) Curvas termogravimétricas TG/DTG da amostra MCM-41/CTMABr não calcinada; b) Curvas termogravimétricas TG/DTG da amostra MCM-41/TDTMABr não calcinada.

As amostras sintetizadas apresentaram as três perdas de massa características do material mesoporoso tipo MCM-41. Esses resultados estão apresentados na Tabela 03.

Tabela 03 - Quantificação das etapas de perda de massa das peneiras moleculares MCM-41/CTMABr e MCM-41/ TDTMABr.

\begin{tabular}{|c|c|c|c|c|c|c|}
\hline \multirow{2}{*}{$\begin{array}{l}\text { Amostra } \\
\text { Evento }\end{array}$} & \multicolumn{3}{|c|}{ Faixa de Temperatura $\left({ }^{\circ} \mathrm{C}\right)$} & \multicolumn{3}{|c|}{ Perda de Massa (\%) } \\
\hline & (I) & (II) & (III) & (I) & (II) & (III) \\
\hline $\begin{array}{c}\text { MCM- } \\
\text { 41/CTMABr }\end{array}$ & $35-138$ & $138-382$ & $382-555$ & 9,67 & 24,57 & 4,50 \\
\hline $\begin{array}{c}\text { MCM- } \\
\text { 41/TDTMAB }\end{array}$ & $33-144$ & $144-356$ & $356-559$ & 9,22 & 25,31 & 4,87 \\
\hline
\end{tabular}

\section{4 - Conclusões}

Os resultados das análises permitiram-nos obter as seguintes conclusões: 
- Os materiais mesoporosos do tipo MCM-41 foram sintetizados com sucesso através do método hidrotérmico, utilizando o brometo de cetiltrimetilamônio e o brometo de tetradeciltrimetilamônio como direcionadores estrutural, a $100^{\circ} \mathrm{C}$ por 120 horas, com ajuste de $\mathrm{pH}$ para a faixa de 9,5-10 a cada 24 horas;

- Os padrões de DRX de todas as amostras apresentaram três picos típicos, um com uma elevada intensidade, atribuída a linha de reflexão do plano (100) e dois outros com menor intensidade atribuídos às reflexões dos planos (110) e (200) característicos da estrutura hexagonal mesoporosa;

- Os espectros de absorção na região do infravermelho dos materiais mesoporosos tipo MCM-41 correspondendo à região espectral entre 4000 e $400 \mathrm{~cm}^{-1}$, apresentam bandas características dos estiramentos assimétricos e simétricos $\mathrm{Si}-\mathrm{O}-\mathrm{Si}$, das vibrações $\equiv \mathrm{Si}-\mathrm{OH}$ ou $\equiv \mathrm{Si}-\mathrm{O}^{-}$. Através dos espectros de FTIR é possível verificar que o processo de calcinação remove todo o material orgânico contido nos materiais, uma vez que os espectros das amostras calcinadas não apresentam a banda na região relacionada ao material orgânico.

Abstract: In this work the mesoporous molecular sieve MCM-41 type was synthesized from different surfactants aimed at obtaining nanostructured materials with catalytic properties and hydrothermal stability for specific applications. The materials were synthesized by the hydrothermal method at $100^{\circ}$ $\mathrm{C}$ for 120 hours, introducing all samples, the synthesis gel the following molar composition: 1.00 surfactant: $4.00 \mathrm{SiO}_{2} ; 1.00 \mathrm{Na}_{2} \mathrm{O}: 200,00 \mathrm{H}_{2} \mathrm{O}$. The resulting materials after calcination at $500^{\circ} \mathrm{C}$ for 2 hours, were characterized by diffraction X-ray (DRX), thermogravimetric (TG / DTG) spectroscopy in the infrared (FT-IR), and surface area by the BET method, The DRX analyzes of the catalysts indicated that the material had hexagonal structure characteristic of mesoporous MCM-41 type material. The TG curve showed that the decomposition occurs in the driver structural materials in temperatures below $550^{\circ} \mathrm{C}$. The samples exhibited variations in the specific surface area, average pore diameter and thickness of the silica wall, due to the surfactants.

Keywords: Molecular sieves; MCM-41; Hydrothermal synthesis; surfactants.

\section{5 - Referências bibliográficas}

BECK, J S; SCHMITT, K. D.; HIGGINS, J. B.; SCHLENKERT, J. L. New Family of Mesoporous Molecular Sieves Prepared with Liquid Crystal Templates. Journal American Chemistrey Society. n. 14, p. 10834-10843, 1992.

FLANIGEN, E. M.; Studies in Surface Science and Catalysis. 1991, 58, 13.

IUPAC Manual of Symbols and Terminology, Pure Applied Chemistry, 31, p 578, 1978; S.I.

KIM, J. M.; KWAK, J. H.; JUN, S.; RYOO, R. Ion Exchange and Thermal Stability of MCM-41. Zeolites, p. 16742-16747, 1995.

KRESG, C. T.; LEONOWICZ, M. E.; ROTH, W. J., VARTULI, J. C. \& BECK, J. S. Nature. 359, 1992. p. $710-712$. 
KRUK, M.; SAYARI, A.; JARONIEC, M., Studies in Surface Science and Catalysis, 129 (2000) 567.

RIGHT, Paul A., HUMPHREY H.P. Yiu, BOTTING, Nigel P. Microporous and Mesoporous Materials. 44-45. Elsevier, 2001, p. 763-768.

SCHUTH, F., Propriedades de superfície e desempenho catalítico de Novos Mesostrucured Óxidos, Ber. Bunsenges. Phys. Chem., 99, p. 1306,1995. 